\begin{tabular}{|c|c|}
\hline Title & $\begin{array}{l}\text { A Di st ri but ed Know edge Net work for Real Wbrl d } \\
\text { Robot Appl i cat i ons }\end{array}$ \\
\hline Author(s) & $\begin{array}{l}\text { Chong, Nak Young; Hongu, Hi r oshi ; Ohba, Koht ar o; } \\
\text { H r rai , Shi geoki ; Tani e, Kazuo }\end{array}$ \\
\hline Citation & $\begin{array}{l}\text { Proceedi ngs of } 2004 \text { I EEE/RSJ I nt er nat i onal } \\
\text { Conf er ence on I nt el I i gent Robot s and Syst ens } \\
\text { ( I ROS 2004): 187- } 192\end{array}$ \\
\hline Issue Date & 2004 \\
\hline Type & Conf er ence Paper \\
\hline Text version & publ i sher \\
\hline URL & ht t p: //hdl . handl e. net /10119/9754 \\
\hline Rights & 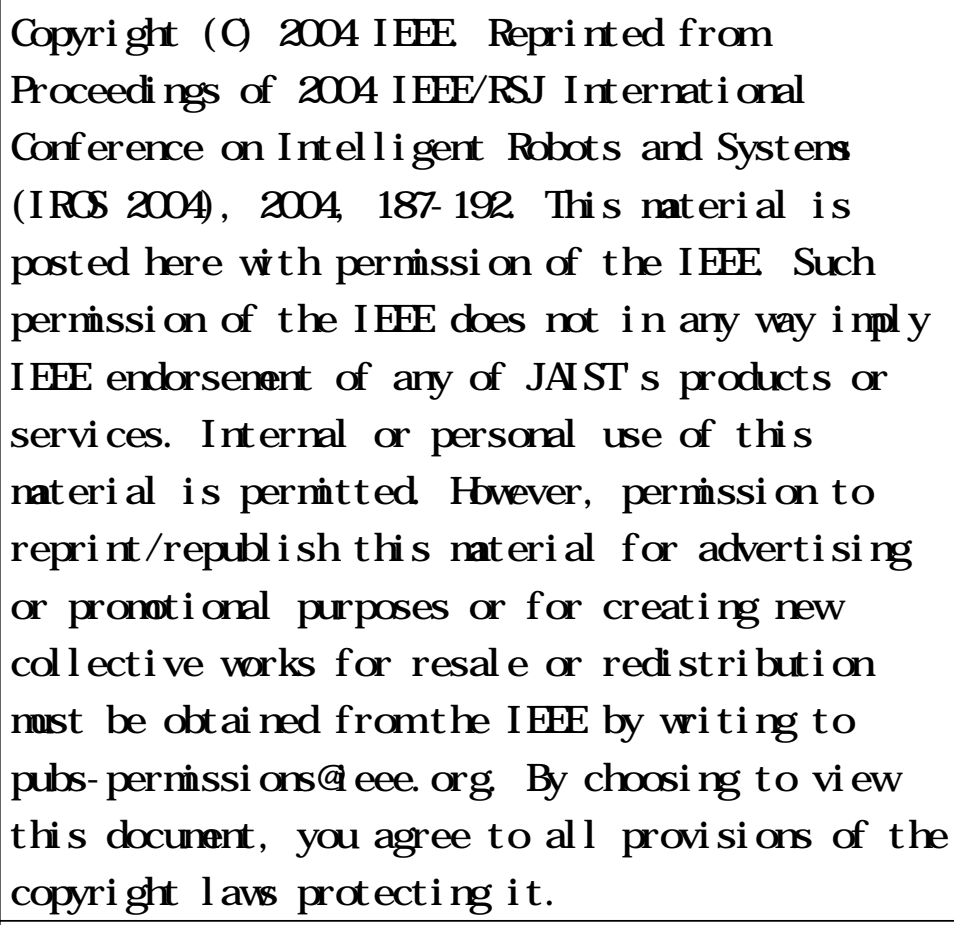 \\
\hline Des & \\
\hline
\end{tabular}

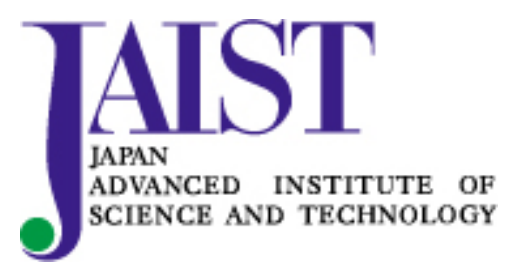




\section{A Distributed Knowledge Network for Real World Robot Applications}

\author{
Nak Young Chong \\ School of Information Science \\ JAIST \\ Tatsunokuchi, Japan \\ Email: nychong@icee.org
}

\author{
Hiroshi Hongu \\ Advanced Engineering Systems \\ University of Tsukuba \\ Tsukuba, Japan \\ Email: h-honguu@aist.go.jp
}

\author{
Kohtaro Ohba, Shigeoki Hirai, Kazuo Tanie \\ Intelligent Systems Institute \\ AIST \\ Tsukuba, Japan \\ Email: k.ohba,s.hirai,tanie.k@aist.go.jp
}

\begin{abstract}
We propose a collaborative knowledge network that we call Omniscient Spaces in the attempt to generate sophisticated robotic behavior with minimal programming effort. New products are manufactured and brought into our daily life everyday. Robots should need a way to easily integrate new products into their existing recognizable environments. Radio frequency identification gains increasing attention to support context and ambient awareness in dynamically changing environments. To solve robot programming difficulties in our environments, the collaborative knowledge network connects heterogeneous knowledge resources to collectively build up the robot's knowledge required to accomplish a task. Specifically, a decentralized knowledge acquisition and task specific integration model is proposed, where the proposed knowledge integrator merges specific knowledge with existing knowledge into a task requiring knowledge. For this, manufacturers put their product data tailored to plan robot motions online and robots may access the data without authorization. In this work, the best possible scenario under current technological limitations is proposed for real world robot applications. A detailed analysis of the knowledge flow model is described. To verify the validity of the proposed model, a test bed is built and table clearing task is performed according to the distributed knowledge management framework.
\end{abstract}

\section{INTRODUCTION}

Even though several humanoid robots have made their successful debut recently and attracted media attention, they are still very far from the popular image of a working robot. It turned out that they still have difficulties in recognition and motion generation in dynamically changing environments such as our daily environments. New products are manufactured and brought into our daily life everyday. This requires new recognition process to keep the robot updated on what is new and application program development to handle those new products. Therefore, end-users should have good knowledge of their robot programming. However, programming robots has proven to be quite an experience and seems almost impossible for ordinary end users. A true autonomous robot with selfrecognition and leaming is still far away due to our current technological limitations.

Therefore, to cope with robot programming difficulties in changing environments, we need to develop an open and adaptive networking environments that seamlessly merge the new products with existing objects and generate product-specific application programs with no additional effort [4]. Building a pervasive computing environment [12] is meaningful in a sense that every entity can be monitored and controlled to some extent. Accordingly, the robot is aware of the object information that can be updated in real-time. Likewise, an architecture for knowledge-based object registration was proposed in computer vision [1]. But the service provided, because of an information management perspective, has been mostly limited to location awareness and resource discovery. Thus, a new interface framework is needed to help the robot build its program automatically in changing environments, whereby it can provide a wide range of services with more sophisticatedly tailored behavior.

Recently, radio frequency identification (RFID) systems gain increasing attention, since the radio frequency eliminates the need for an optical line of sight and transmits a relatively large amount of information [6], [11]. Moreover, it can be used for the bi-directional information flow. Thus, if we embed an RFID tag in every object and localize the tagged object, this can be a powerful solution for the robot environment recognition. Following this approach, we proposed a new paradigm of the interaction through RFID allowing robots to easily identify and activate tagembedded home appliances [2]. We generalize this concept to include common household chores. Our proposed distributed knowledge network that connects distributed product databases and the robot can be a support infrastructure for automatic integration of heterogeneous knowledge sources to build an application program and download it to the robot. In this work, a decentralized knowledge acquisition and task specific integration model is proposed, where the proposed knowledge integrator merges specific knowledge with existing knowledge into a task requiring knowledge. An experimental observation of the proposed system is reported.

\section{KNOWLEDGE IN A DISTRIBUTED ENVIRONMENT}

In many robotic applications with the model-based approach, it is common for the robot end-user (or programmer) to pre-describe the characteristics of the environment being recognized before any application program is developed, but this may not apply where the environments are not so well structured and are changing such as our daily environments. It is apparent that the changing environment 


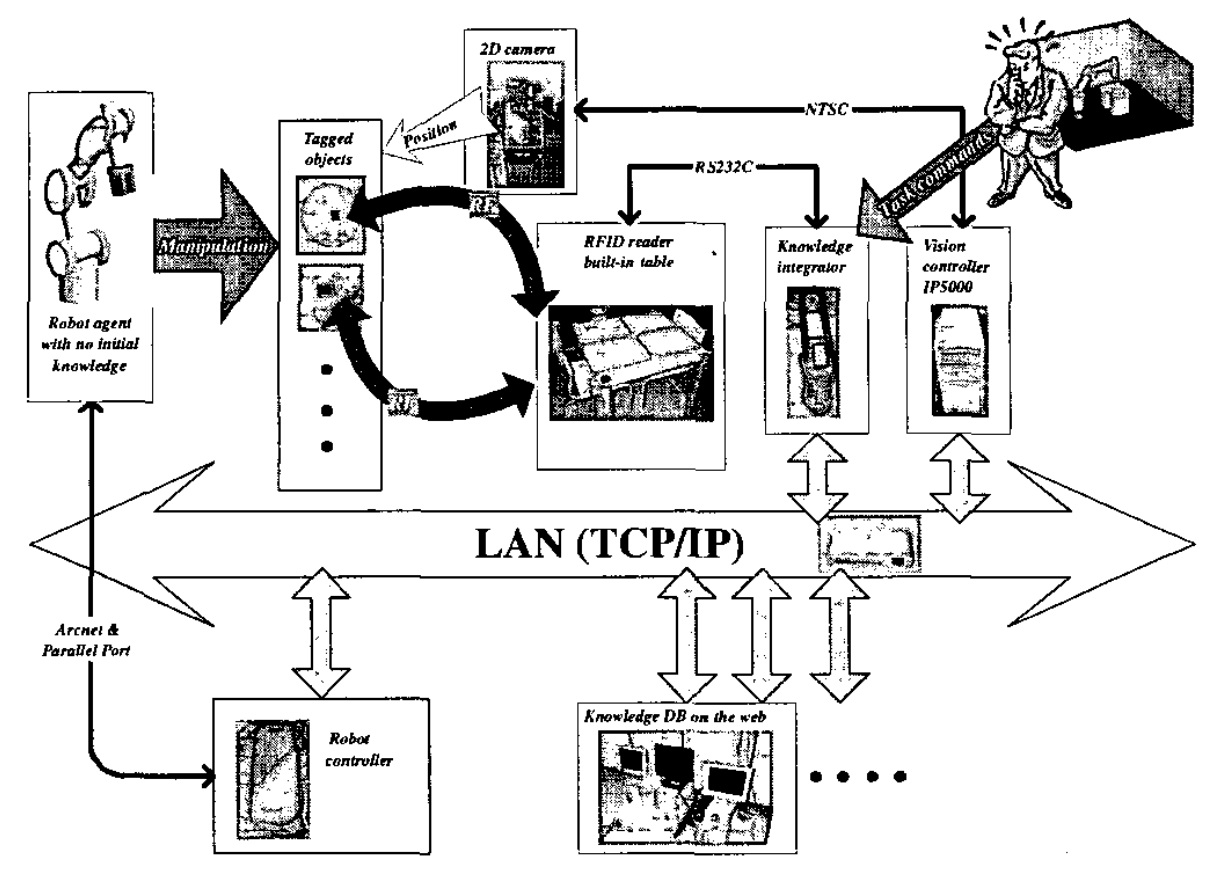

Fig. 1. A knowledge flow model.

can not be predicted and robots need constant updates on the new knowledge within the environment. In such environments, the enormous amount of knowledge, that the end-user needs to pre-input, may reside and accordingly a large capacity storage device is needed.

Therefore, to cope with the complexity of knowledge description and comprehension and the limited storage capacity, we proposed knowledge distributed robot control framework [4]. Thanks to recent advances in information and communication technology, efficient storage and retrieval of data and information became possible. Any information geographically distributed across multiple locations can be collected using a variety of technologies. Therefore, the communication between the robot and an object in the networked environment must consider the data gathering process of the object. No single recognition system proves adequate for diverse robot applications. Using an integrated recognition system such as tag-based vision, robots can understand the context of the environment more effectively.

Assuming that an integrated sensing and computing tag is embedded into every single object in a distributed environment, the robot becomes fully aware of its environment. Then, moreover, the object information stored in the tag can draw out robot motion by giving a direction on how to deal with it. In the knowledge distributed network, the object (product) manufacturer can describe and input the product knowledge into the product and/or upload it to the public network. Diverse products of a variety of different manufacturers may exist in our environments. Practically, we should make an attempt to standardize input formats and foster mutual compatibility of all databases. Also, the product data should be tailored to merge into robot application programs easily.

\section{A DECENTRALIZED MODEL, FOR KNOWLEDGE FLOW}

In this section, we address a decentralized model for knowledge flow control to validate the proposed distributed knowledge network. As one of the common household chores, table clearing is considered toward practical use of the robot assistant in the house. (See Fig. 1.) A robot is commanded to pick up dinnerware from the table and stack it up on the nearby cart or put it in the trash basket (or other pre-specified positions) until all dishes have been cleared. In the task, the robot is taught to recognize various objects such as dishes and bowls and learn about those objects, whereby it can make appropriate decisions about the task. The robot autonomously creates an overall task sequence, choose an object pick-up strategy, and determine the object return position.

It should be stressed that the robot has no preprogrammed knowledge about the object and the task. Environmental knowledge will help the robot develop the competence for the task. Our scenario proposed here is designed to support the use of decentralized knowledge base model for common kinds of application programming. (See Fig. 2.)

1) The task is commanded to the knowledge integrator PC.

2) The knowledge integrator collects information using RFID devices. The information includes the serial number of the objects, the location where the objects are to be stacked in the cart or other retum positions, 


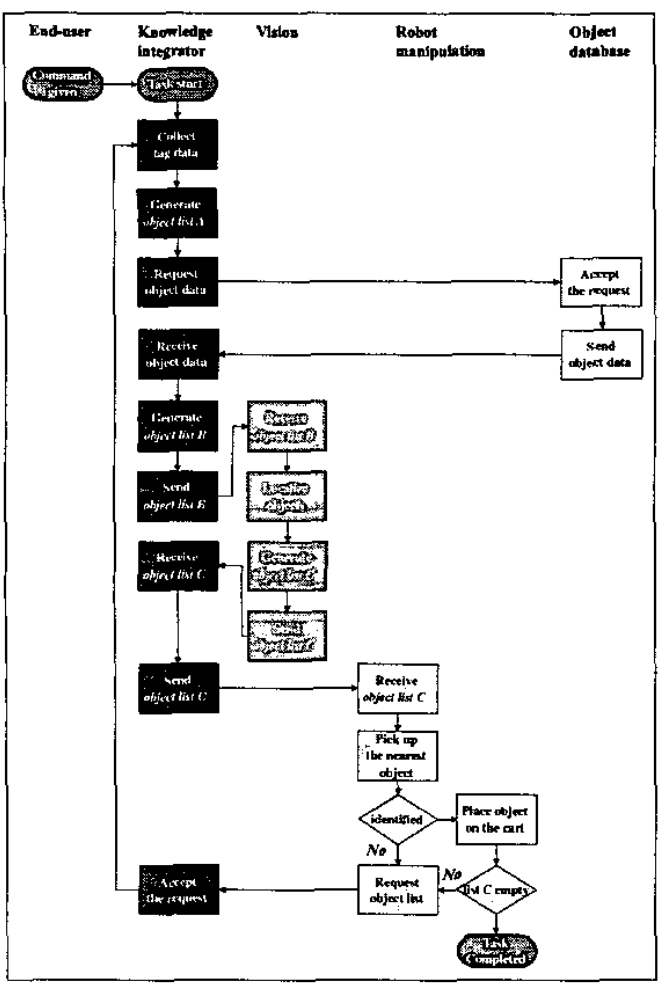

lijg. 2. Flowchan for knowledge generation and integration.

and the specific web site address where more details about the object are given.

3) Based on the information, the knowledge integrator creates the knowledge list (what we call List $A$ in this work) of each object. The task is considered completed if the list is empty.

4) The knowledge integrator accesses the corresponding web address and acquires the necessary information (what we call List B) required to pick up the object such as the grasping position and orientation and the image template associated with the tag ID.

5) The vision system detects the location of the objects through template matching. The List $C$ is generated.

6) The robot picks up the nearest object to avoid possible collisions.

7) The knowledge integrator collects RFID data again and checks out the object $\mathrm{ID}$ taken away at the previous step. This will help the robot identify the ID of the grasped object.

8) The robot stacks up the objects on the cart. Some objects can be perceived as having the same shape. However, the tag data allows the robot to separate the objects properly.

9) The system returns to step 2) and repeats steps 2) through 8 ) until the knowledge list becomes empty.

\section{EXPERIMENTS}

A prototype system is implemented using the framework of distributed knowledge networks.

\section{A. Software}

The World Wide Web (WWW) is a distributed hypermedia system available through the Intemet [14]. The object knowledge source written in Extensible Markup Language (XML) as shown in Fig. 4 is uploaded to the WWW server. This enables the knowledge integrator to acquire knowledge from diverse sources geographically distributed. The knowledge integration software is implemented in EusLisp [5] and is developed for the data acquisition and management to integrate and send or receive data to/from distributed locations. EusLisp is an integrated programming system for the research on intelligent robots based on Common Lisp and object-oriented programming. Object location sensing and robot manipulation softwares are implemented in $\mathrm{C}++$ and TCP/IP network protocol is used on LANs. More details can be found in [8].

\section{B. Object location sensing}

There have been many proposals for indoor location awareness. Those systems may differ from one another by the target application [7]. Since the RFID system is not designed for location sensing [3], [10], [13], the vision system is used together with RFID [9]. A ceiling mounted CCD camera is used to get the correct position of the objects on the table. The Hitachi IP5000, a fast image processing system on a half-size PCI board, is inserted into the controller PC running Linux to connect an NTSC camera and video output monitor. The board is equipped with 40 video memories of $512 \times 512$ pixels and provides plenty of fast image processing functions in hardware.

The basic location sensing strategy is to utilize an object database associated with the object tag ID. The image template of each object is stored in the database. The list of the objects to be detected is generated by the table built-in RFID system and this requires only a finite number of searches in the database. Based on the List B received from the knowledge integrator by the plain text communication through a TCP/IP socket, the input camera image is compared with the database of image templates and the center point is detected in the camera image frame. In the experiment, the image template is stored in vision control PC and RFID tag information is used to bring out the proper template. Then, the coordinates are transformed into corresponding Cartesian coordinates with respect to the robot end-effector coordinate frame. These coordinates are added to the List $B$ to generate the List $C$ which is sent back to the knowledge integrator.

\section{Robot manipulation}

Robot manipulation is hierarchically organized: a planner part that generates the position and orientation command and the gripper on/off signal based on the detailed knowledge in the List $C$ and the robot control part. In the collaborative knowledge network, robot manufacturers 
provide their control software. Thus, the end-user will not need to develop control softwares for the robot. From that standpoint, a Mitsubishi PA-10 robot is controlled by the company's motion control CPU board in the robot controller PC running Windows XP. The motion control CPU board and the servo driver are communicated at a control frequency of $2 \mathrm{msec}$ through the high-speed serial communication ARCNET. The Takano Bearing Model RH707 is attached as an end-effector and connected to the same controller PC through parallel digital $\mathrm{V} / \mathrm{O}$.

The robot receives the List $C$ from the knowledge integrator by the plain text communication through a TCP/IP socket connection. The received list is then sorted in increasing order based on the sum of squares of $x$ and $y$ coordinates. This enables the robot to detect the nearest object in the list. The end-effector orientation (roll, pitch, and yaw) in the first data set is sent to the robot. Then, with the orientation, the robot moves to the position in the list and closes the end-effector. Picking up the object, the robot requests the object list on the table again to the knowledge integrator and compares the new list against the old list. The grasped object should not be included in the new list, since it is out of the read range of the RFID reader. Moving to the return position, the robot opens the end-effector to place the object in position. The index number in the list points the locations on the cart or other return points. The position data of the stack location can be stored in the database of the cart, but for simplicity they are stored in the robot controller in the experiment. If a multiple objects are to be stacked up, the z-axis (height) data of the stack location is updated automatically according to the number of objects stacked in that location. This process is repeated until the list is empty.

\section{RESUlts}

Tables I through III show the List $A, B$, and $C$, respectively, generated during a test run of the experiment. The List $A$ includes the number of objects on the table, the object ID, the URL address, and the disposal index followed by a zero reserved for future expansion. We assumed that the table height was known (or could be known by its tag data) and the objects were symmetrical. The knowledge networks helped the robot collect what the individual system knows in a geographically distributed environment. Thus, based on actual data transmission from the environment, the robot can determine the possible grasp position around the object. For instance, the radius data and the height data of the object was given from the object database and the center point data from the vision system. (See Fig. 3.) Thus, the second list, List B, additionally includes the grasp point information of the object such as the $z$-axis coordinate, the deviation from the center of the object, and the end-effector orientation. The final list, List $C$, includes $x$ and $y$ coordinates of the object center point with respect to the robot end-effector frame. The robot coordinates in the List $C$ are defined in Fig. 5.

A physical test bed of the proposed system was built in October 2003. No systematic trouble has been found in

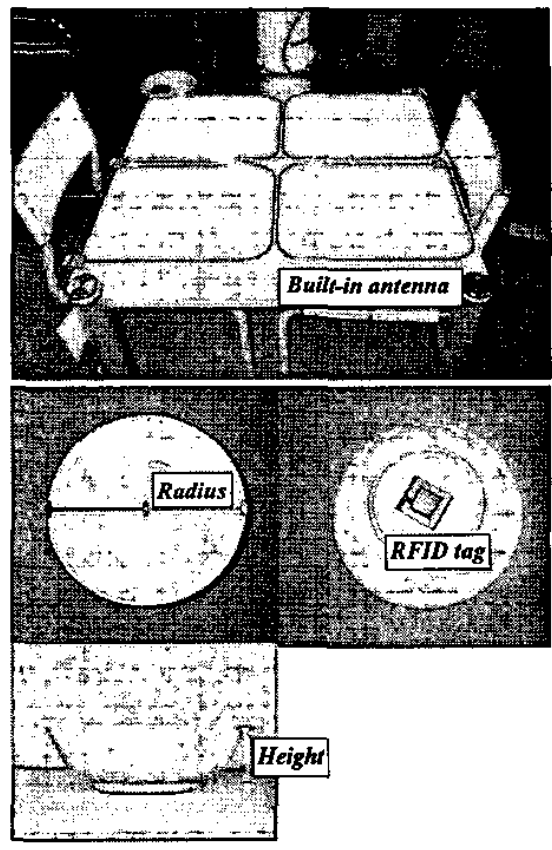

Fig. 3. Table built-in RFID antenna and tagged object.

\begin{tabular}{|c|}
\hline 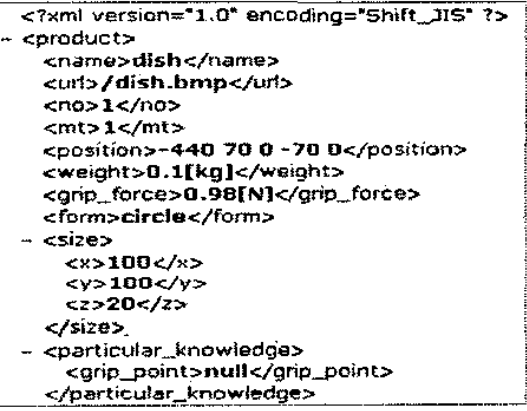 \\
\hline
\end{tabular}

Fig. 4. A sample description of object knowledge written in XML.

the demonstration test since then. Fig. 6 shows the test bed used in the development of knowledge networks and Fig. 7 the snapshots of successive robot motions during testing. The robot in the test bed responded properly to the new object incidentally added to the table during the testing process without any additional computation or knowledge request. We were convinced that no ad hoc process was needed. The networked RFID infrastructure assisted the robot in understanding rapidly changing environments. Likewise, it helped improve vision-based location sensing on the table.

\section{CONCLUSION}

We proposed a new design of distributed knowledge networks to deploy easy-to-use robot systems into our daily life with minimal programming effort. The test bed has been built at AIST in Tsukuba, Japan to facilitate the 
TABLE I

A KNOWLEDGE List A FROM RFID DATA ACQUISITION

\begin{tabular}{|llll|}
\hline $5 \longleftarrow$ number of objects & & \\
49312f... & http://tagvvv.a09.aist.go.jp/di... & 7 & 0 \\
c9302f... & http://tagserver.a09.aist.go.jp/go... & 4 & 0 \\
c5302f... & http://tagvvv.a09.aist.go.jp/di... & 1 & 0 \\
c8302f... & http://md117022.a09.aist.go.jp/bo... & 2 & 0 \\
d1302f... & http://tagserver.a09.aist.go.jp/cu... & 3 & 0 \\
$\uparrow$ & $\uparrow$ & $\uparrow$ & $\uparrow$ \\
ID & URL & Disposal & Buffer \\
& & index & \\
END & & & \\
\hline
\end{tabular}

TABLE II

A KNOWLEDGE List $B$ FROM WER SERVERS

\begin{tabular}{|c|c|c|c|c|c|c|c|c|}
\hline \multicolumn{9}{|c|}{$5 \leftarrow$ number of objects } \\
\hline $49312 f . .$. & http://tagvvv.a09.aist.go.jp/di... & -440 & 70 & 0 & -70 & 0 & 7 & 0 \\
\hline c9302f... & http://tagserver.a09.aist.go.jp/go... & -285 & -20 & 90 & 0 & -90 & 4 & 0 \\
\hline c5302f... & http://tagvvv.a09.aist.go.jp/di... & -440 & 70 & 0 & -70 & 0 & 1 & 0 \\
\hline $\operatorname{c8302f} \ldots$ & http://md1 17022.a09.aist.go.jp/bo... & -452 & 60 & 0 & .28 & 0 & 2 & 0 \\
\hline d1 $302 f . .$. & http://tagserver.a09.aist.go.jp/cu... & -385 & 30 & 0 & -5 & 0 & 3 & 0 \\
\hline$\uparrow$ & $\uparrow$ & $\uparrow$ & $\uparrow$ & $\uparrow$ & $\uparrow$ & $\uparrow$ & $\uparrow$ & $\uparrow$ \\
\hline ID & $U R L$ & $z$ & Dev & Yaw & Pitch & Roll & $\begin{array}{l}\text { Disposal } \\
\text { index }\end{array}$ & Buffer \\
\hline END & & & & & & & & \\
\hline
\end{tabular}

TABLE III

A KNOWLEDGE List $C$ FOR ROBOT MANIPULATION

\begin{tabular}{|c|c|c|c|c|c|c|c|c|c|c|}
\hline \multicolumn{11}{|c|}{$5 \leftarrow$ number of objects } \\
\hline $49312 \mathrm{f} . .$. & http://tagvvv.a09.aist.go.jp/di... & 180 & 197 & -440 & 70 & 0 & -70 & 0 & 7 & 0 \\
\hline c $9302 f \ldots$ & http://tagserver.a09.aist.go.jp/go... & 544 & -285 & -285 & -20 & 90 & 0 & -90 & 4 & 0 \\
\hline c5302f... & http://tagvvv.a09.aist.go.jp/di... & 520 & 168 & .440 & 70 & 0 & -70 & 0 & 1 & 0 \\
\hline$c 8302 f .$. & http://md1 17022.a09.aist.go.jp/bo... & 111 & -212 & -452 & 60 & 0 & -28 & 0 & 2 & 0 \\
\hline d] $302 f . .$. & http://tagserver.a09.aist.go.jp/cu... & 549 & -173 & -385 & 30 & 0 & -5 & 0 & 3 & 0 \\
\hline$\uparrow$ & $\uparrow$ & $\uparrow$ & $\uparrow$ & $\uparrow$ & $\uparrow$ & $\uparrow$ & & $\uparrow$ & $\uparrow$ & $\uparrow$ \\
\hline$I D$ & $U R L$ & $x$ & $y$ & $z$ & Dev & Yaw & Pitch & Roll & $\begin{array}{l}\text { Disposal } \\
\text { index }\end{array}$ & Buffer \\
\hline
\end{tabular}

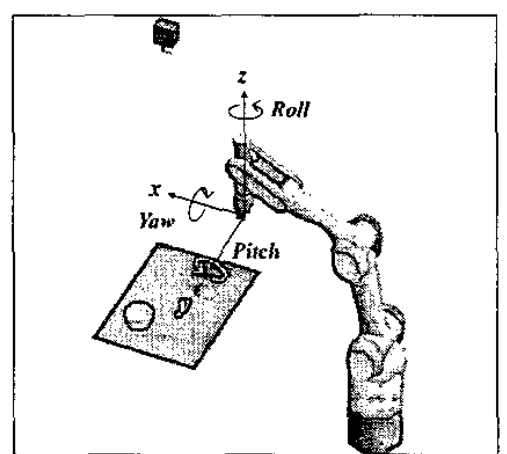

Fig. 5. Coordinate definition in the knowledge list.

study of spatial knowledge acquisition and integration for automatic robot behavior generation. A collaborative management of distributed knowledge resources were stressed. As a view to possible implementations, detailed experimental conditions and results were described. Practically there are many ways to program a robot. In this work, without any significant human intervention, the robot cleared the dinning table autonomously that is an extremely useful goal one may want to achieve for real world robotic applications.

\section{ACKNOWLEDGMENT}

The authors would like to thank Manabu Miyazaki, Koji Takemura, and Kenichi Ohara for their assistance with this project at AIST.

\section{REFERENCES}

[1] M. Boukraa and S. Ando, An Invariance-Based Object Registration Architceture Using Electronic Tags and XML. Proc. 1st Int. Conf. on Information Technology and Applications, paper 87-5, Bathurst, Australía, 2002.

[2] N.Y. Chong and K. Tanje, Dependable Maniputation Based on RFID. Proc. Int. Symp. on Robotics and Automation, pp. 330-335, Toluca. Mexico, 2002.

[3] N.Y. Chong, H. Hongu, and K. Tanie, Object Localization in Omniscient Spaces. Proc. Int. Conf. on Advanced Robotics. pp. 118123, Coimbra, Portugal, 2003. 

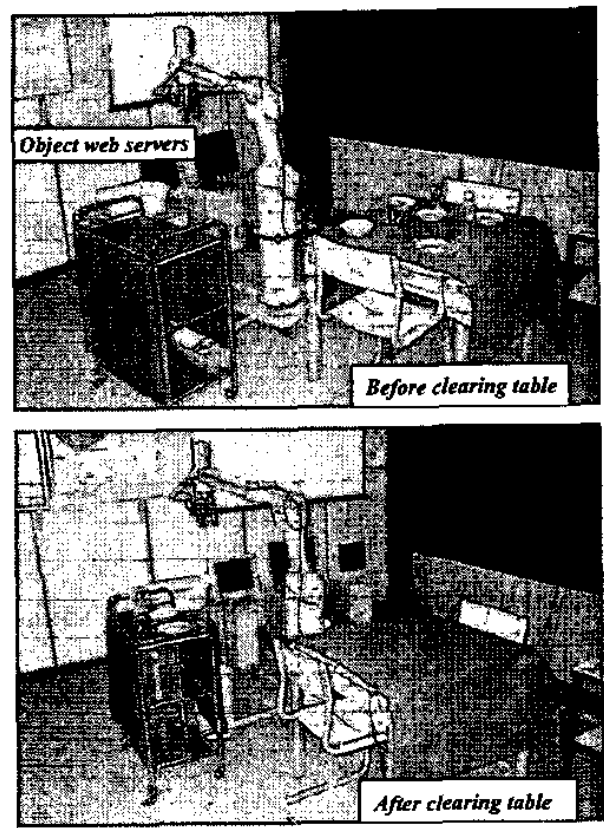

Fig. 6. Table clearing in the test bed.

[4] N.Y. Chong, H. Hongu. M. Miyazaki, K. Takemura, K. Ohara, K. Ohba, S. Hirai, and K. Tanie, Robots on Self-Organizing Knowledgc Networks. Proc. IEEE Int. Conf. on Robotics and Automation, New Orleans, LA. U.S.A., 2004.

[5] EusLisp, http://www:dh.aist.go.jp/ t.matsui/eus/euslisp.html.

[6] K. Finkenzeller, RFID Handbook: Radio-Frequency Identification Fundamentals and Applications, John Wiley \& Sons Lid., 1999.

17] J. Hightower and G. Borriello, Location Systems for Ubiquitous Computing. IEEE Computer Magazine, pp. 57-66, 2001.

$181 \mathrm{H}$. Hongu A Study on Knowledge Distributed Rohot Systems Using IC Tags. Master's Thesis, Advanced Engineering Systems. University of 'Tsukuba, 2004 (in Japanese).

(9) R. Horaud, F. Domaika and B. Espiau, Visually Guided Object Grasping. IEEE Transactions on Robotics and Automation, pp. 525 532. Vol, 14 , No. 4, 1998

[10] M. Kim, T. Takeuchi, N.Y. Chong, 3-D I Localization Using a 3-axis Orthogonal Anlenna. Proc. JSME Conf. on Robotics and Mechatronics, 2004.

1111 Y. lee and P. Sorrells, microlD $13.56 \mathrm{MHz}$ RFID System Design Guide, Microchip Technology Inc., 2001

[12] MIT Project Oxygen, htp://oxygen.Ics.mit.edw/.

[13] I Siio User Position Detection Using RFID Tags. Information Processing Society of Japan Human Interface Special Interest Group Note 00-HI-88. pp. 45-50, 2000 (in Japanese).

[14] The World Wide Web Consortium, http:/www:w3.org/.

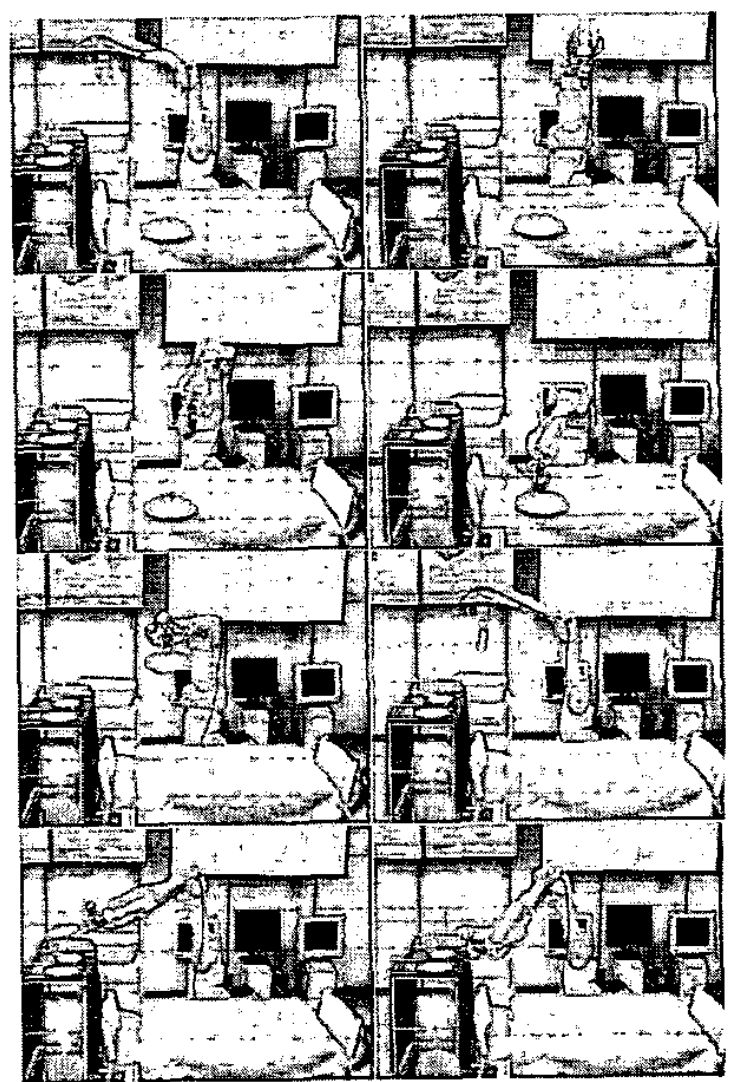

Fig. 7. Snapshots of successive motions of the robot. 